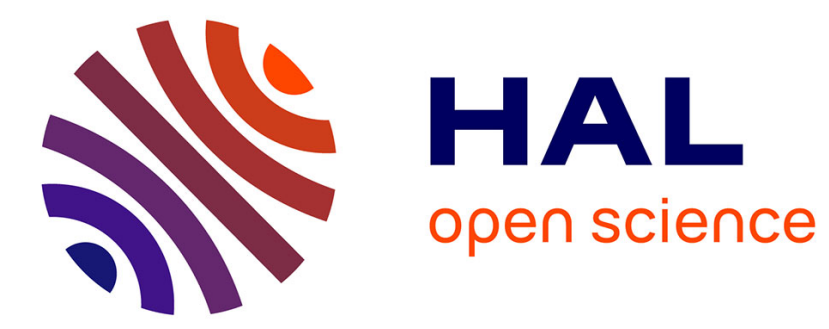

\title{
A discharge coordinate detection system
}

B. Bommeli, C. Frei

\section{- To cite this version:}

B. Bommeli, C. Frei. A discharge coordinate detection system. Revue de Physique Appliquée, 1977, 12 (12), pp.1861-1867. 10.1051/rphysap:0197700120120186100 . jpa-00244414

\section{HAL Id: jpa-00244414 https://hal.science/jpa-00244414}

Submitted on 1 Jan 1977

HAL is a multi-disciplinary open access archive for the deposit and dissemination of scientific research documents, whether they are published or not. The documents may come from teaching and research institutions in France or abroad, or from public or private research centers.
L'archive ouverte pluridisciplinaire HAL, est destinée au dépôt et à la diffusion de documents scientifiques de niveau recherche, publiés ou non, émanant des établissements d'enseignement et de recherche français ou étrangers, des laboratoires publics ou privés. 


\author{
Classification \\ Physics Abstracts \\ $77.50(52.80)-07.50$
}

\title{
A DISCHARGE COORDINATE DETECTION SYSTEM
}

\author{
B. BOMMELI and C. FREI \\ Section de Physique, University of Geneva, \\ 1211 Geneva 4, Switzerland
}

(Reçu le 31 mai 1977, accepté le 5 septembre 1977)

\begin{abstract}
Résumé. - L'appareillage que nous décrivons permet d'enregistrer les coordonnées de l'endroit sur les électrodes où ont lieu les décharges électriques. Ces décharges sont générées à une fréquence de répétition élevée, dans un intervalle qui est rempli d'un liquide isolant et qui est de petite dimension par rapport à celle des électrodes. La distribution des lieux de décharges, ainsi que nous l'avons relevé à l'aide de cet appareillage, dépend des conditions auxquelles les décharges sont soumises. On peut rencontrer, soit une distribution totalement aléatoire, s'étendant sur toute la surface des électrodes, soit une localisation des décharges dans une ou plusieurs régions des électrodes.
\end{abstract}

\begin{abstract}
A device is described which makes it possible to record the coordinates of the spots of recurrent electrical discharges. These discharges are produced between electrodes which are separated by a small gap filled with an insulating liquid. Conditioning effects occurring in the liquid dielectric are presumed to modify the discharge pattern. An initial survey with our detection system indicates that under certain circumstances a completely random distribution of the discharges becomes possible. However, we can also observe discharge patterns, where the discharge spots are localized at one or several regions on the electrodes.
\end{abstract}

1. Introduction. - When electric discharges are applied in a repetitive manner between two electrodes separated by a thin film of an insulating liquid, we notice that the location of the disruption shifts in an irregular manner. The experimental conditions are such that the opposing electrode surfaces, between which the discharges occur and which do not necessarily have to be flat, are parallel to each other, the separation being typically between 10 and $100 \mu \mathrm{m}$. The surfaces extend over distances of several centimeters. A tension either of less than a hundred volts or up to several hundred volts may be applied to cause breakdown of the dielectric liquid. Any insulating liquid may serve as a dielectric. The sequence of the discharges is timed in such a way that once the dielectric has broken down, a given discharge current it maintained during an interval of chosen length. Then a break follows, the length of which may be varied from a few microseconds to a few milliseconds, these limits being the same as for the discharge duration. When the voltage is applied again, the following breakdown will usually occur at another place on the electrodes. The shifting of the discharge spot may be brought into relationship with the conditioning effect on breakdown strength due to the consecutive discharges [1]. The distribution of the discharge coordinates should be a source of valuable information with respect to the mechanism of breakdown.
In this context, we would like to recall that our understanding of electric discharges in a liquid dielectric is still in the early stages [2]. One of the reasons is to be found in the enormous experimental difficulties in investigating the discharge process. As a consequence of the short time scale and the very smallness of the space in which the discharge events occur the number of physical quantities which can be measured is small as compared with the number of the physical quantities which are involved in a complete description of the phenomenon. Therefore, any supplementary information of an experimental nature which can be retrieved from the discharge process would be welcomed as a possibility of checking the various ideas which have been put forward concerning the discharge mechanisms.

In parallel with the scientific aspects of the measurements of the spatial discharge distribution there exists a technological interest which is related to the procedure of machining with electrical discharges. In electro-discharge machining, metal is removed from a workpiece, connected as one of the electrodes, by the erosive action of the arc discharge. The arc represents the final stage of the discharge which starts with the breakdown of the liquid phase. It is an essential feature of this process that the spots of consecutive discharges shift from one place to another. When a condition exists whereby the discharges become sta- 
tionary, the metal surface to be machined will deteriorate : in which case it would be useful to be able to determine these detrimental conditions [3].

In this paper we give a detailed description of a device capable of recording the coordinates of a sequence of discharges. It consists of a segmented electrode system, electronic circuitry for sensing the flow of the discharge current, and equipment for the acquisition of the data. The principle of coordinate detection underlying the device is much the same as that for spark chambers in nuclear physics. However, as put into practice there remains little resemblance, since the discharge medium, a liquid, has very different electrical properties compared to a gas, and since the device serves a different purpose.

In order to illustrate the performance of the coordinate detection system and to show the type of information provided, a few results are given. The details of the results obtained with this equipment will be accounted for in a separate publication.

2. The electrodes. - In order to determine the coordinates of a discharge between adjacent electrodes both electrodes are built up of a set of thin metal plates, each one being electrically insulated from the neighbouring plates. The two electrode sets are mounted in such a way, that the plates of the upper electrode are oriented in a direction perpendicular to that of the plates of the lower electrode (Fig. 1). Therefore, if current detectors identify the plate of each set, which carries the discharge current, the Cartesian coordinates of the discharge can be determined. All that is required

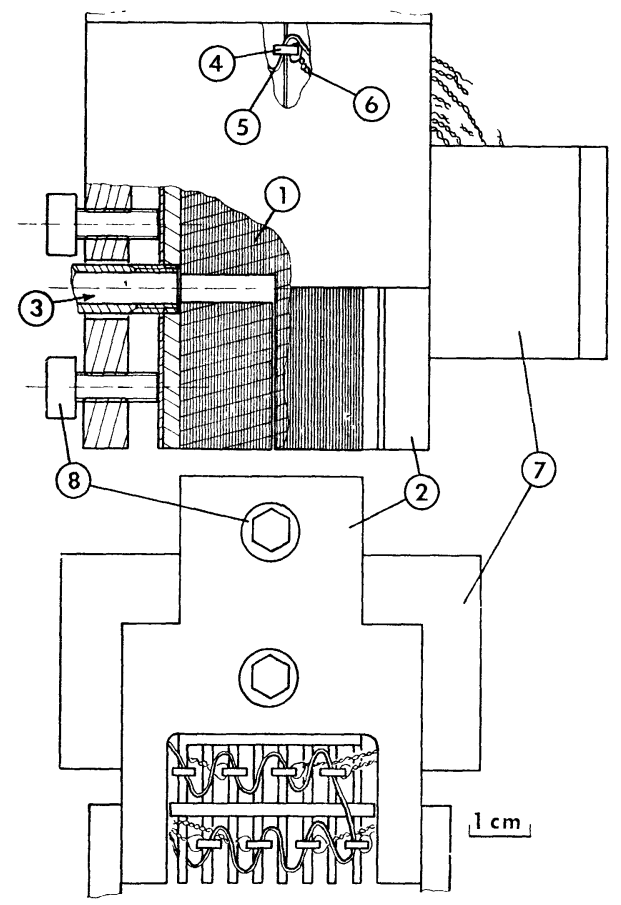

FIG. 1. - Schematic view of the electrode system : 1. electrode plates, 2. support, 3 . central injection hole for dielectric liquid, 4. ferrite core, 5 . wire for polarizing current, 6 . signal carrying secondary wires, 7. connectors, 8 . clamping screws; the gap between upper and lower electrode is not to scale. is to multiply the plate ordinal number with the distance between consecutive plates.

There is a danger that discharge ignition might be modified as a result of field distortion at the edges of neighbouring plates, due to the fact that we have replaced a planar electrode by a set of thin plates. Although the field distorsion is quite small, since all plates of an electrode are maintained at the same potential, it is advisable to choose the insulating layers as thin as possible. After testing different materials, we selected sheets of mica as being most satisfactory. Discharges cause a localized increase of temperature up to the melting point of the electrodes. Organic compounds were not retained as insulating material because they tend to retract and, thus, to favor short circuits between adjacent plates. Mica, on the contrary, remains intact up to the electrode surface, where, under the action of the discharges, it is removed at the same rate at which the metal is removed by erosion. Despite the fact that mica responds to our requisites, there is a lower limit to the thickness of the insulating layer that can be chosen in view of the risk of too frequent short circuits between neighbouring plates. We consequently selected a $0.03 \mathrm{~mm}$ condensor type quality of mica. Given a typical electrode gap of $25 \mu \mathrm{m}$, the distortion of the electric field remains thus quite small. Since the size of erosion craters lies between 50 and $100 \mu \mathrm{m}$, the ultimate stages of the discharge process should be influenced very little by the presence of the insulating material.

The short-circuiting of adjacent plates is thought to be caused by the resolidification of the metal which is ejected out of discharge craters near the border of a plate. Resolidification of metal splashed around craters is a familiar picture to those who examine electrode surfaces with a microscope. It should also be noticed that there is a resemblance between these short circuits and those occuring between opposite electrodes. We have explained the category of low resistance short circuits as being produced by tiny metal bridges [4].

After several tests with different plate thicknesses, the final assembly of the electrodes consisted of stacking alternatively metal sheets of $0.5 \mathrm{~mm}$ and mica insulation layers of $0.03 \mathrm{~mm}$. Copper was chosen for the first electrode and steel for the second. Each electrode is composed of 60 plates which form a stack of approximately $30 \mathrm{~mm}$. On crossing two stacks of this size, we obtain an active electrode surface which has the form of a square of $30 \mathrm{~mm}$ by $30 \mathrm{~mm}$. The electrodes are mounted on a spark erosion machine (Charmilles D 10) which provides automatic control of the discharge gap (Fig. 2).

Before measurements can be made, the surfaces of the two electrode stacks must be made parallel to each other. Prior to assembly of the stacks, the surfaces are carefully machined on a milling machine. The remaining surface roughness as well as possible deviations from parallelism can be corrected for after the elec- 


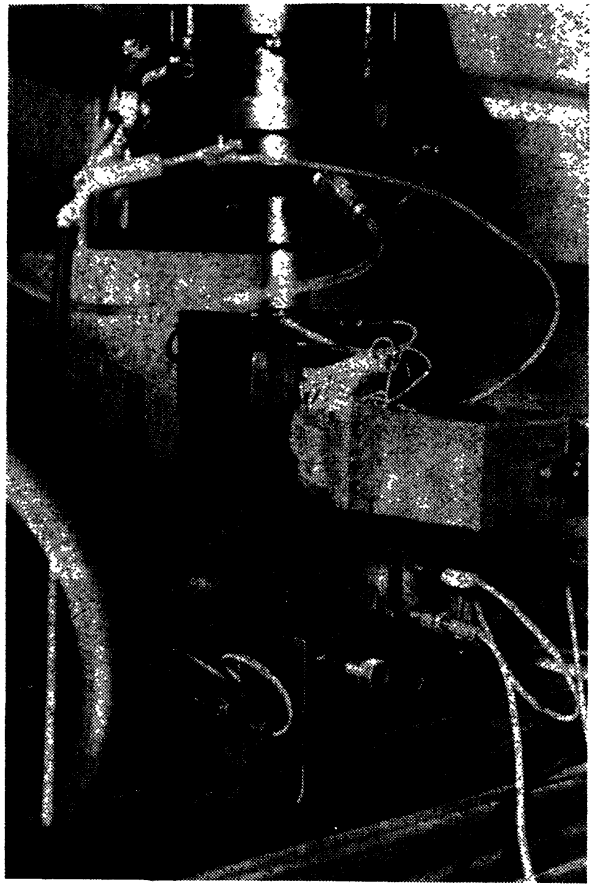

Fig. 2. - Electrode assembly mounted on spark erosion machine Charmilles D 10; the two electrodes are separated from each other for visual inspection.

trodes have been mounted on the spark erosion machine. For this purpose, the machine is operated according to its design when it is used for machining metal pieces. The discharges occur preferentially on those parts of the electrode surfaces which are closest. After a short period of spark machining the surplus metal is removed.

In order to check the parallelism in a few instances, the two electrodes were polished simultaneously by

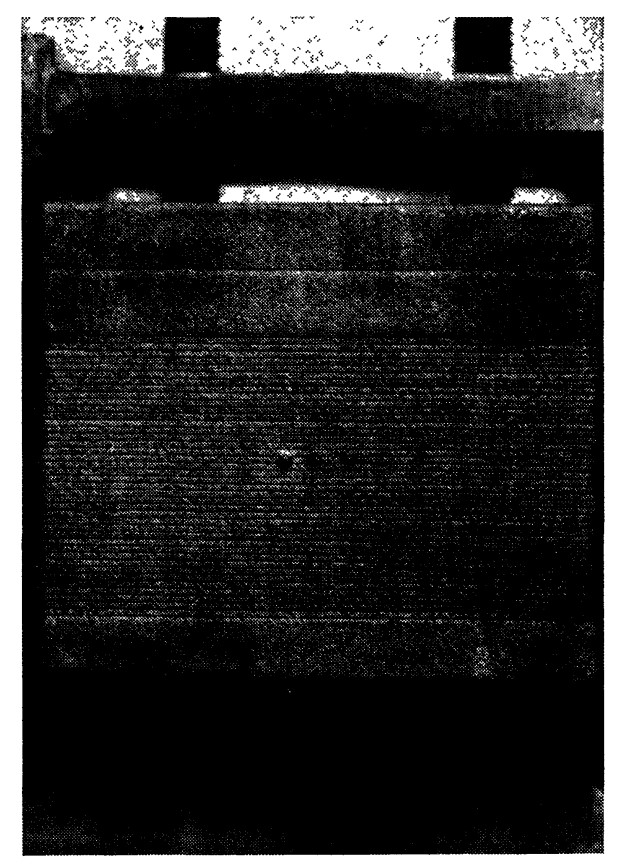

FIG. 3. - Specimen showing attack of the electrode surface by discharges; thickness of narrow plates : $0.5 \mathrm{~mm}$, of large plates : $5 \mathrm{~mm}$. inserting between them a highly parallel metal plate. The mechanical guidance of the electrodes in the direction perpendicular to their surfaces is guaranteed to a few $\mu \mathrm{m}$. A similar degree of precision of parallelism follows from the uniformity of the surface attack when the electrodes are partially polished in the above mentioned configuration by means of the metal plate and a polishing agent. On a microscopic scale deviations from parallelism are of course also present due to the erosive action of the discharges. The surface roughness depends on the pulse energy and varies typically between a few tenths of a $\mu \mathrm{m}$ to some $\mu \mathrm{m}$.

The diameter of the discharge craters on the electrode surface would limit ultimately the coordinate resolution (in the electrode plane) of the detection system. However, in our case the plate thickness is larger than the diameter of the craters (Fig. 3) and hence the resolution is determined by the plate thickness.

3. The current detectors. - Each one of the plates of the two sets of electrodes are equipped with current detectors. These identify the pair of plates which conduct the current associated with a particular discharge. The current detectors consist of ferrite core transformers mounted on the wires which connect each plate with the pulse generator's output. Whilst the primary winding is identical with the wire connecting the plate to the generator, the secondary winding is made of a single turn of wire. The information concerning the discharge coordinates is carried as an analogous signal from the detector to the electronic unit which converts it into a digital code.

Although the impulse generator produces a constant current level the rise of the discharge current is subject to considerable fluctuations. The voltage pulse induced in the secondary winding varies, consequently, within wide limits and is, therefore, unsuitable for proper discrimination from noise. The difficulty is avoided by integrating the induced voltage and by choosing a ferrite material with small hysteresis and rapid saturation (Fig. 4). Thus, we obtain a signal of roughly constant level. The pulse amplitude remains the same for whatever discharge current is chosen, provided it is above the threshold level which is necessary to saturate the ferrite.

Integration by a simple $\mathrm{RC}$-network gives rise to a voltage difference across the capacity $C$, such that :

$$
U=\frac{1}{R C} \int\left[U_{\text {ind }}(t)-U_{\text {diode }}-U\right] \mathrm{d} t .
$$

With $R C$ sufficiently large, $U$ becomes small by comparison to $U_{\text {ind }}$ and may be neglected in the bracket. Similarily, we neglect the small voltage drop across the diode, $U_{\text {diode }}$, which is in series with the $R C$ network. The diode serves the purpose of encoding the plate number and will be referred to later. The integral of the induced voltage depends on the initial and the final value of the magnetic flux $\varphi=B S, B$ correspon- 


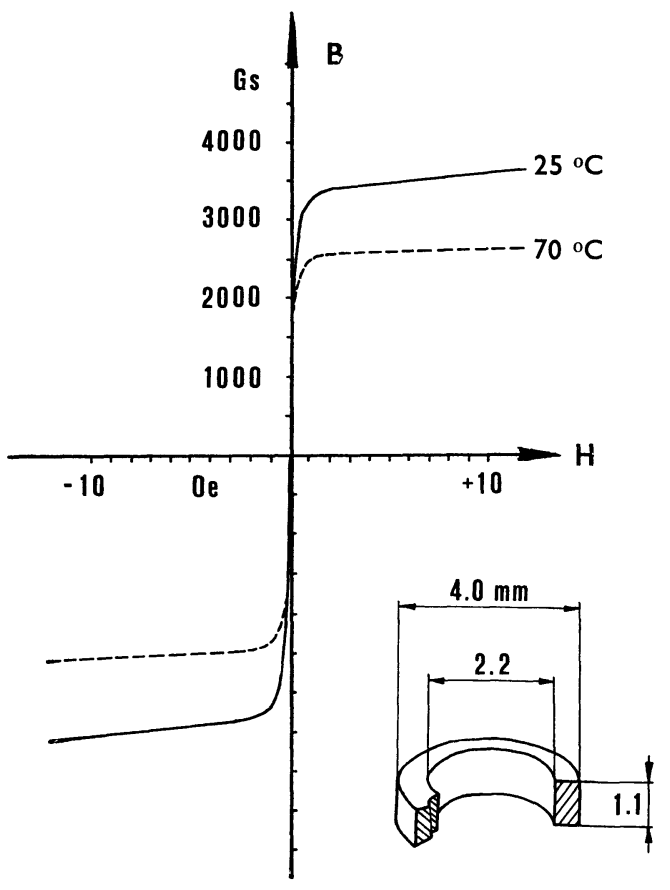

Fig. 4. - Magnetization curve of ferrite (Philips $3 \mathrm{E} 2$ ) at $25^{\circ} \mathrm{C}$ and at $70{ }^{\circ} \mathrm{C}$ and size of core.

ding to the induction of the magnetic material and $S$ to the core section. When running the transformer core from field $H=0$ into saturation and back again, no reproducible behaviour can be expected in so far as the state of magnetization at $H=0$ is extremely sensitive to small variations of the residual field, to temperature effects on hysterisis etc. To resolve this difficulty, we polarize the ferrite cores by means of a constant current carried on a separate wire which is passing through all the ferrite rings of the array. The polarity of the polarizing current is such that the cores are saturated in the reverse direction. Thus in order to produce a signal output the discharge current must first compensate the polarization current; then, an additional fraction of an ampere is sufficient to saturate the cores. The voltage integrated on the capacitor becomes approximately :

$$
U \approx 2 \frac{B_{\mathrm{sat}}(T) \cdot S}{R C} .
$$

The polarizing wire, common to all ferrite cores, gives rise to a coupling between the cores. Another contribution to coupling stems from the close packing of the detectors and from their wiring. A high polarizing current suppresses to a large degree the perturbations due to these coupling effects : unless the induced currents equal the polarizing current, they are unable to change the state of saturation of the cores and to produce more than a spurios output.

The principal cause of fluctuation of the current signal is the temperature dependence of the saturation value of induction $B_{\text {sat }}$ of the ferrite. As can be inferred from the curves in figure 4 , one should expect a decrea-

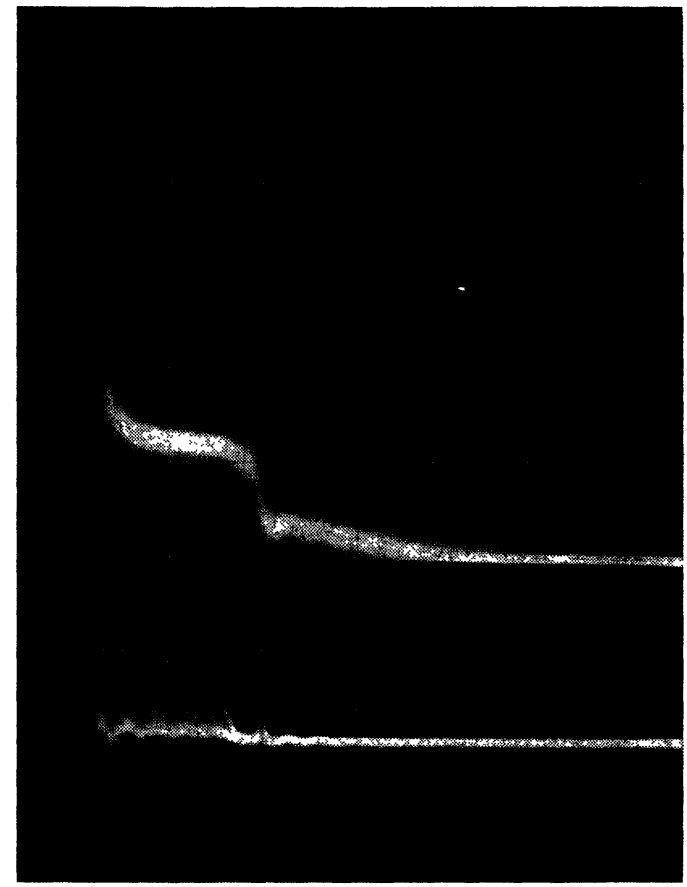

FIG. 5. - Oscillogram of discharge voltage (upper trace $20 \mathrm{~V} /$ division) and of integrated voltage signal (lower trace 0.5 $\mathrm{V} /$ division), from the ferrite core of an electrode plate; time scale : $5 \mu \mathrm{s} /$ division. The superposition of traces when the discharge current passes through other plates shows the noise level.

se of almost one third of the voltage when the temperature rises from ambient to $70^{\circ} \mathrm{C}$. Such temperature changes occur as a consequence of hysteresis losses in the cores, when one core receives several current pulses consecutively. The dispersion of the signal amplitude must be kept within limits, such that the distance from the noise level is sufficiently large to allow for correct discrimination (Fig. 5). This is done by keeping within a given maximum rate of the repetition frequency of the discharges, as will be mentioned further on.

4. The circuitry. - The signals originating from the 60 plates of each electrode enter, prior to integration, a diode matrix which encodes their ordinal number into a 6 bit binary number. A block diagram of the circuit can be found in figure 6. By adopting this pro-

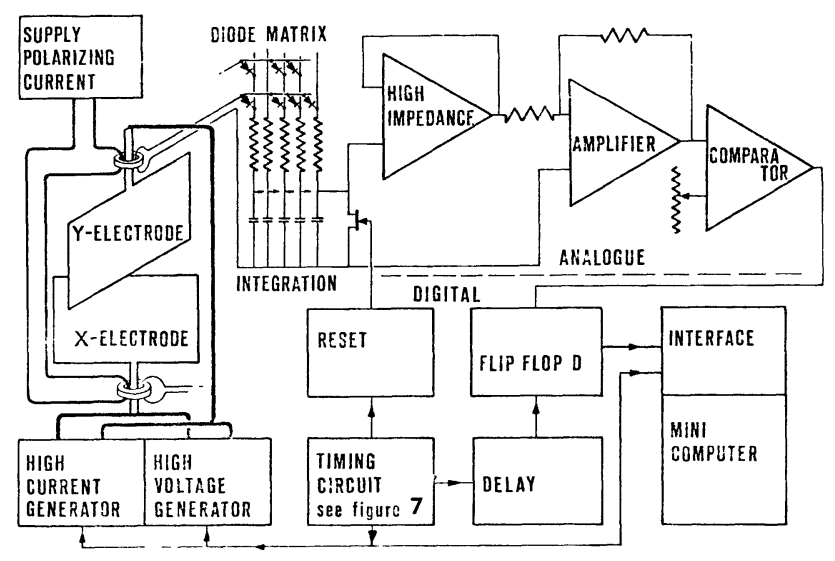

FIG. 6. - Block diagram of coordinate detection system. 
cedure right at the beginning of electronic processing, the number of parallel circuits is limited to 12 ; one circuit for every bit. Simultaneously the diodes prevent the integrating capacitors from discharging. It is at this stage that the information about the coordinates of the discharge spot is first stored. Subsequently a high input impedance section, followed by a slight voltage amplification, transmits the signal to a comprator. The threshold of the comparator is adjusted midway between the highest noise and the lowest pulses originating from discharges. The adjustment is carried out under working conditions, since the noise emanates partly from the high frequency components of the discharge current. As a matter of precaution, the wiring which connects the electrodes with the electronic unit consists of twisted pairs of conductors.

In conformity with TTL logic, the signal is now memorized in a stack of flip flops. At this stage the information is ready to be transferred into the computer via an interface module. The $x$ and $y$ coordinates of a discharge, each available as a 6 bit binary number, are put together to form a computer word. Since the words are made up of 16 bits, 4 bits remain unused. Those 4 bits may be employed for the identification of the discharge type or for the implementation of other operations.

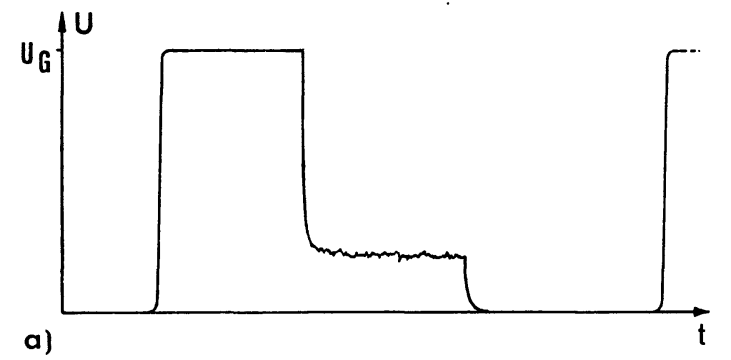

b)

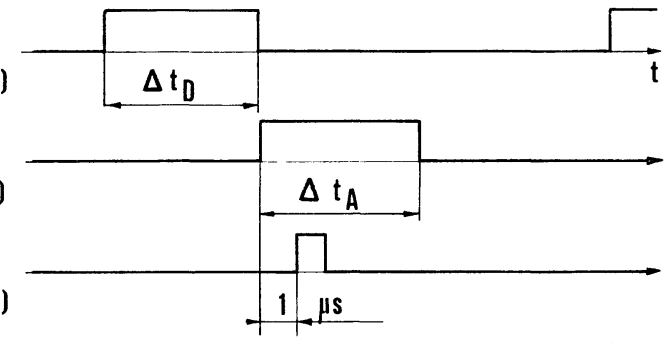

e)

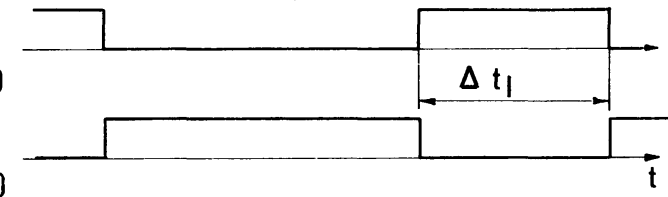

FIG. 7. - Timing sequence referring to a period lasting from one discharge to the next; $a$ ) potential between electrodes ; $b)$ to $f$ ) binary logic signals ; $b$ ) time-lag of breakdown ; c) arc discharge duration; $d$ ) readout of discharge coordinates ; $e$ ) pulse interval duration and reset command signal ; $f$ ) signal commanding the discharge current generators; 2 generators working in tandem are capable of delivering from 4 to $32 \mathrm{~A}$ at a maximum voltage of $450 \mathrm{~V} ; c$ ) and $e$ ) can be chosen within 4 and $1600 \mu \mathrm{s}$, variable in steps of a factor of 2 .
In order to be able to store the signal of the following discharge, the voltage of the integrating capacitor is reset at zero by means of a FET-transistor which is commanded according to the timing sequence displayed in figure 7.

A readout signal, also shown in figure 7, is produced shortly after occurence of breakdown. It causes the flip flops to read the binary encoded discharge coordinates stored in the integrating capacitors and asks the computer, via the interface module, to initiate the data acquisition programme.

5. Data processing. - The large number of discharges which are produced within a short period of time elicit an equally large mass of coordinate values. Since, at the outset, we expected that their distribution contains a dominant statistical component, and since its nature was totally unknown at that time, an individual processing of each coordinate value was thought more appropriate than some kind of analogue averaging procedures, for instance. With this implication in mind, the coordinate detection system was built so as to present the coordinates as binary numbers. In this way the data can be fed into a computer, which alone can resolve in an efficient and easily adaptable way the problem of evaluating a large volume of measurements. To this purpose we use a mini-computer (Hewlett Packard $2114 \mathrm{~A}$ ) with $8 \mathrm{~K}$ words of memory and a word length of 16 bits.

In essence, the computer can be employed in two different ways : either the discharge coordinates are stored in the computer memory in the sequential order in which they become available - in this case the data will be processed at a later stage - ; or the logical and arithmetic operations which must be implemented in order to obtain the desired presentation of the results are executed progressively as the data arrive. The advantage of the first method resides in the fact that all information about a measurement series is maintained in the computer. If necessary, this information can be transferred to a permanent carrier, a tape, for instance. The decision concerning the kind of presentation which best summarizes the collected data can be left to a later stage. It is evident that the original amount of information must be reduced to what is relevant for a description of the discharge pattern. At the outset, the best procedure of reduction is not known with certainty and depends on the results obtained.

The other method of processing the information, mentioned above, offers the advantage of practically unlimited storage capacity of any volume of data deemed necessary. As the incoming data are immediately converted into a correlation scheme, a distribution of the discharge displacements, or into something similar, the memory space required for recording the data equals the space which the scheme of the chosen representation occupies in the computer. This depends on the desired resolution and amounts normally to a 
few hundred computer words. By comparison with this method, the number of events, which can be stored in our computer model, according to the procedure mentioned at the first place, is limited to respectively 3000 or 6000 , depending on whether or not additional parameters are recorded besides the discharge coordinates. It would, therefore, seem advantageous to employ the second method of data processing, as soon as the question of the most appropriate presentation of the results has been solved. However, a further aspect needs to be considered, namely the rate at which the data can be processed. The second method limits this rate so severly that preference was given to the more cumbersome two step procedure, that is : first data storage, followed by data processing. Whenever the statistics require more measurements that can be stored during one acquisition cycle, the acquisition process is repeated again and again and the results accumulated until the statistical requirements are fulfilled. The limitation of the data acquisition rate, when discharge coordinates are recorded according to the first method, has its origin in an excessive increase of the temperature of the ferrite cores of the current detectors. The maximum rate of the repetition frequency of the discharges we have been using was close to $80 \mathrm{kHz}$. When other parameters besides the discharge coordinates are recorded simultaneously, their time of conversion from analogue to digital value in addition to the acquisition time of the computer reduces the speed below the limit due to the current detectors.

6. Results. - This section presents some initial results, the purpose being to communicate a few essential features of the distribution of discharges in a small gap filled with a liquid dielectrique - use was made of a light fraction of paraffin oil (Chevron EDM 71). Also, it illustrates some of the various possibilities of representing the measurements.

In a first approach our measurements were limited to one coordinate only. The distribution of the difference of the $x$-coordinate between consecutive discharges is exemplified in figure 8 by two rather extreme cases : the circles belong to a distribution of almost triangular shape and the crosses correspond to a distribution strongly peaked for small displacements, $\Delta x$. The point at $\Delta x=0$ which lies outside the triangular shape may be explained by the presence of the thicker plates on the border of the electrode. Allowance for a similar effect ought to be made also for the second distribution. The electrode used for these preliminary measurements is the one shown in figure 3. Later, when working in two dimensions, we use the set of electrodes which are composed of 60 equally thick plates of $0.5 \mathrm{~mm}$.

A triangular shaped distribution is obtained when the discharges shift in a completely random fashion on the rectangular electrode surface. Evidence that such is indeed the case can be obtained immediately

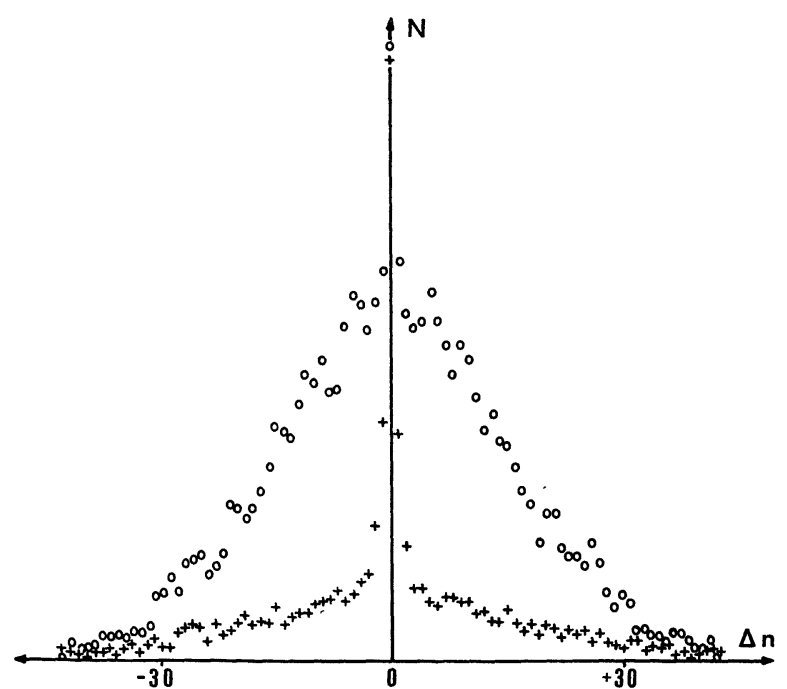

FIG. 8. - Distribution of the $x$-component of the displacements of consecutive discharges with: $\Delta t_{\mathrm{A}}=25 \mu \mathrm{s}, \Delta t_{\mathrm{I}}=100 \mu \mathrm{s}$, $I=32 \mathrm{~A}, U_{\mathrm{G}}=80 \mathrm{~V}$; o stationary, + slowly moving dielectric liquid. Rather than the difference $\Delta x$, the experiment records the difference of the plate ordinal numbers : $\Delta \mathrm{n}$. These are not quite equivalent, since the plates are not all of the same thickness (electrode as on Fig. 3).

from a recording of the coordinates of consecutive discharge spots, as the example in figure 9 illustrates. Although randomness is by no means a general characteristic, the fact of its occurrence precludes for instance the idea that the breakdown spot of a discharge is conditioned by the previous discharge.

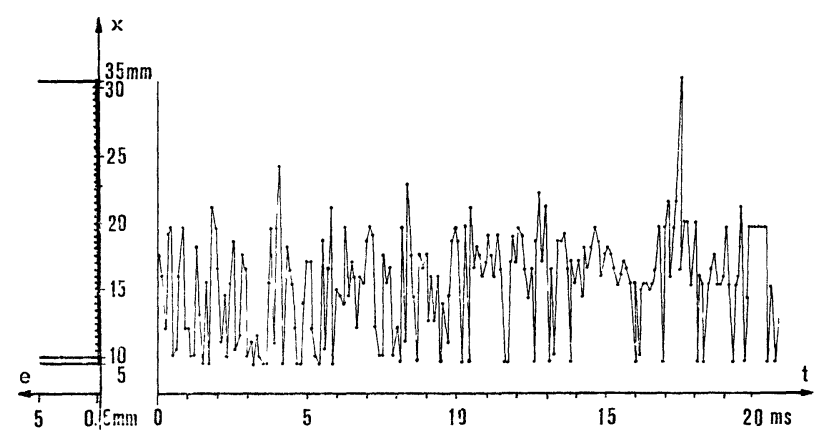

FIG. 9. - $x$-coordinate of discharge spots as a function of time. Discharge parameters : $\Delta t_{\mathrm{A}}=4 \mu \mathrm{s}, \Delta t_{\mathrm{I}}=100 \mu \mathrm{s}, I=16 \mathrm{~A}$, $U_{\mathrm{G}}=80 \mathrm{~V}$ (for thickness e of electrode plates refer to Fig. 3).

The possibility of a concentration of discharges in a limited region of the electrodes has been born out by the peaked distribution, shown in figure 8 . Frequently distributions appear with a pair or with several pairs of peaks lying symmetrically on both sides with respect to $\Delta x=0$. They point to the existence of two or more active sites between which the discharge spot alternates. Direct evidence for this is obtained when the measurements are accomplished in two dimension. Owing to the procedure of storing sequentially the coordinates in the computer memory, we can reproduce the spots of a series of discharges directly on a 
$x y$-plotter. Thus we get patterns like that of figure 10 . Upon following the pen of the plotter when it traces each spot on the graph, one obtains a vivid and lively impression of what is happening in reality.

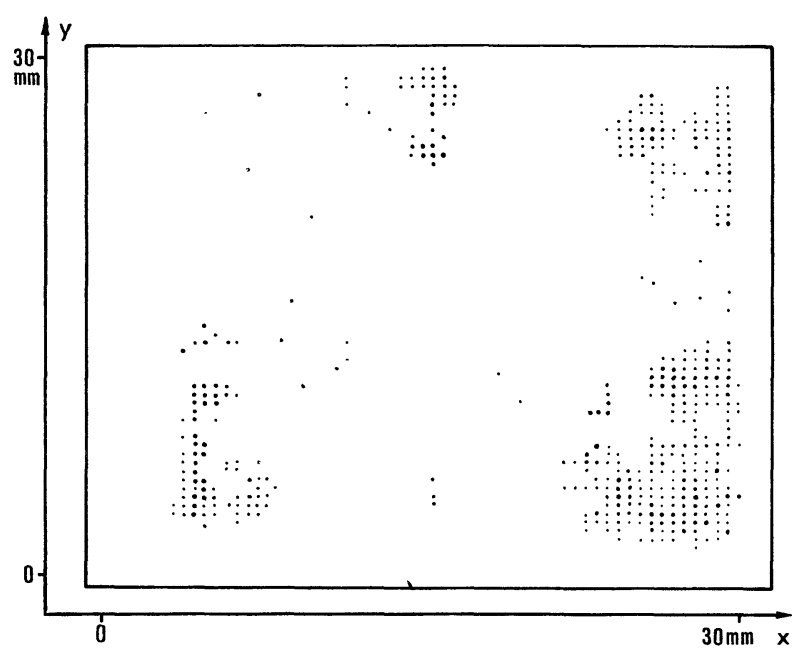

FIG. 10. - Spatial distribution of discharges in the $x y$-plane ; each point represents the position of one or several discharges with : $\Delta t_{\mathrm{A}}=2 \mu \mathrm{s}, \Delta t_{\mathrm{I}}=100 \mu \mathrm{s}, I=32 \mathrm{~A}, U_{\mathrm{G}}=80 \mathrm{~V}$. The electrodes consist of 2 stacks of 60 plates of $0.5 \mathrm{~mm}$. The example chosen illustrates the occurrence of several regions of discharge activity which happen to be located close to the edge of the electrodes.

A step further in the analysis of the discharge patterns is accomplished by relating the shifts with other discharge parameters. Figure 11 shows, by way

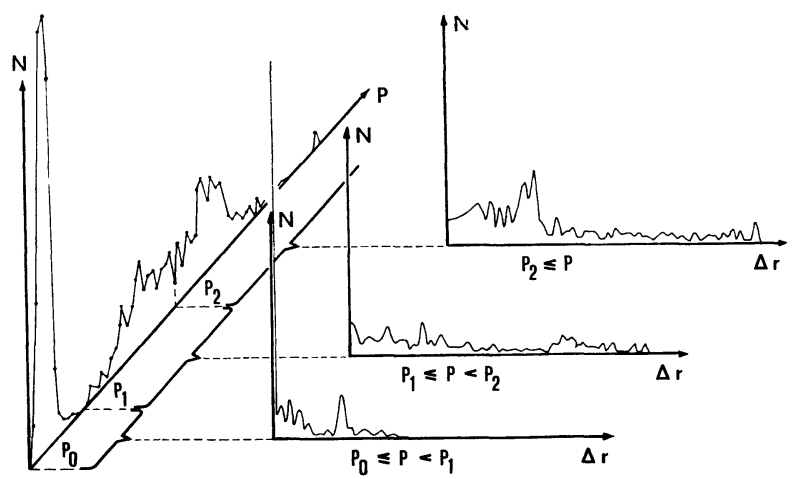

FIG. 11. - Distribution of the absolute values of the displacements of consecutive discharges : $\Delta r=\left(\Delta x^{2}+\Delta y^{2}\right)^{1 / 2}$. The distributions are split according to the discharge type as determined by the discharge arc voltage (discrimination levels at $\mathrm{P}_{1}$ and $\left.\mathrm{P}_{2}\right)$. Discharge parameters : $\Delta t_{\mathrm{A}}=12 \mu \mathrm{s}, \Delta t_{\mathrm{I}}=100 \mu \mathrm{s}$, $I=12 \mathrm{~A}, U_{\mathrm{G}}=350 \mathrm{~V}$. Same type of electrodes as in figures 2 and 10. of example, the distribution of the distances between the spot of a discharge and that of the following in relation to the arc voltage of the following discharge. It demonstrates the existence of a correlation between discharge types and the different kinds of distribution patterns.

7. Conclusion. - This survey of a few typical results shows that the spatial distributions of recurrent discharges may display a large variety of patterns : they range from a completely random type of distribution to the kind where consecutive discharge spots become strongly correlated. The discharges may cover the electrode surface entirely or only partially. In the latter case the active surface may be split into separate areas. The discharges move freely within these areas or may jump from one area to another. The active sites on the electrode surface are constantly changing: they shift slowly from one place to another ; if occa. sionally they split into separate areas, they may, later on, merge again; they can also grow or diminish. Under special circumstances they concentrate on a small spot, whereby the discharges degenerate into a stationary sustained arc. When this happens it causes considerable damage to the electrode surface due to locally high dissipation of energy. The investigation of the conditions leading to such a situation should provide useful information when electrical discharges are put into operation for machining purposes.

As regards the mechanism of discharges in a contaminated dielectric liquid our coordinate detection system is capable of providing new insight : we would like to attract notice to the occurence of random distributions of the discharge spots which means that the events under consideration are uncorrelated. We conclude, therefore, that the breakdown of an electrically stressed dielectric is independent of the instantaneous perturbation of the liquid or electrode surfaces caused by the secondary effects of the discharges. Short-lived emission products of any kind : light, waves, molecular or atomic species do not enhance the probability of ignition of the following discharges. The factors leading to breakdown must be searched for among the more stationary physical causes.

Acknowledgments. - We would like to acknowledge gratefully the financial support given to this project by the Ateliers des Charmilles S. A., Geneva.

\section{References}

[1] Frei, C., Perréard, E. and Peter, M., Helv. Phys. Acta 45 (1972) 778.

[2] Proc. 5th Int. Conf. on Conduction and Breakdown in Dielectric Liquids ; (Delf University Press) 1975.
[3] Balleys, F., Bommeli, B. and Frei, C., Annals CIRP 22/1 (1973) 53.

[4] Balleys, F., Frei, C. and Manzin, E., Fertigung 5/73 (1973) 147. 